Rapid Communications

\title{
Spatially-resolved Observation of Glow Discharge Plasma for Atomic Emission Spectrometry
}

\author{
Yoshitaka ZeNITANI and Kazuaki WAGATSUMA ${ }^{\dagger}$ \\ Institute for Materials Research, Tohoku University, 2-1-1 Katahira, Sendai 980-8577, Japan
}

\begin{abstract}
An imaging spectrograph equipped with a CCD detector was employed to measure two-dimensional emission images from a glow discharge plasma in atomic emission spectrometry. The emission images at $\mathrm{Zn} \mathrm{I} 334.50 \mathrm{~nm}$ for a zinc sample and at $\mathrm{Cu}$ I $324.75 \mathrm{~nm}$ for a copper sample could be obtained. Their emission intensities were not uniform in the radial direction of the plasma region but became weaker at larger distance from the central zone. The two-dimensional distribution would result from a spatial variation in the excitation efficiency of the plasma and thus provide useful information for understanding the excitation processes occurring in the plasma.
\end{abstract}

(Received April 14, 2008; Accepted April 25, 2008; Published May 10, 2008)

\section{Introduction}

Glow discharge optical emission spectrometry (GD-OES) is widely employed in surface analysis as well as bulk composition analysis as a direct analytical method of solid samples. ${ }^{1,2}$ GDOES can provide depth profiles of the sample composition without ultra-high vacuum atmosphere and with a little sample pretreatment; thus, it has been applied for industrial purpose such as on-site analysis. ${ }^{3,4}$ In conventional measurement systems for GD-OES, emission signals from the glow discharge excitation source are observed from the axial direction of the plasma and thus collected onto the entrance slit of the spectrometer with a point-focused lens. Therefore, this optical alignment cannot yield information on the spatial distribution of the emission signal in the radial direction of the plasma, whereas the emission intensity is integrated over the overall region of the plasma.

It can be considered that a two-dimensional (2D) image of the spectrum emitted from the glow discharge plasma would give useful information not only for determining the experimental conditions in the analytical application but for studying the excitation processes occurring in the plasma. For this purpose, an interference filter may be employed to disperse the emission signals; however, they are not suitable for measuring emission spectra comprising lots of spectral lines, because of its bad spectral resolution of more than $10 \mathrm{~nm}$. Gamez et al. have first reported on spatially-resolved elemental analysis in GD-OES by using an imaging spectrometer system. ${ }^{5}$ However, few papers concerning the 2D spectral images of the glow discharge plasma were published, principally due to lack of spectrometers enabling 2D observation having good spectral resolution. This paper indicates 2D images of the emission signal in the radial direction of the glow discharge plasma, when copper and zinc emission lines are observed by using a two-dimensionally imaging spectrometer having a spectral resolution of less than $1 \mathrm{~nm}$.

† To whom correspondence should be addressed.

E-mail: wagatuma@imr.tohoku.ac.jp

\section{Experimental}

Figure 1 shows the block diagram of the measuring system employed. A glow discharge lamp was made in our laboratory according to the original model of Grimm, ${ }^{6,7}$ where the inner diameter of the hollow anode was $8.0 \mathrm{~mm}$ and the distance between the anode and cathode sample was adjusted to be 0.2 $0.4 \mathrm{~mm}$. Emission signals from the glow discharge lamp, which were observed from the axial direction of the plasma, were conducted through a collimator optics onto the entrance slit of an image spectrograph (Model 12580, BunkoKeiki Corp., Japan), and the emission image was then dispersed and detected on a charge-coupled device detector (SensiCam QE Model, PCO Imaging Corp., Germany), where the 2D image of a particular emission line could be observed in the radial direction of the plasma. A $2 \mathrm{D}$ image obtained by $5 \times 5$ pixels corresponded to an actual sample area of $0.1 \times 0.1 \mathrm{~mm}^{2}$. The spectral resolution was $0.1-1.0 \mathrm{~nm}$ depending on the slit width.

Pulsated discharge voltages were applied to the glow discharge lamp with a high-speed high-voltage amplifier (HEOPT-B60L1, Matsusada Precision Inc., Japan), which was controlled with a function generator (Model 4502, Kikusui Corp., Japan). The

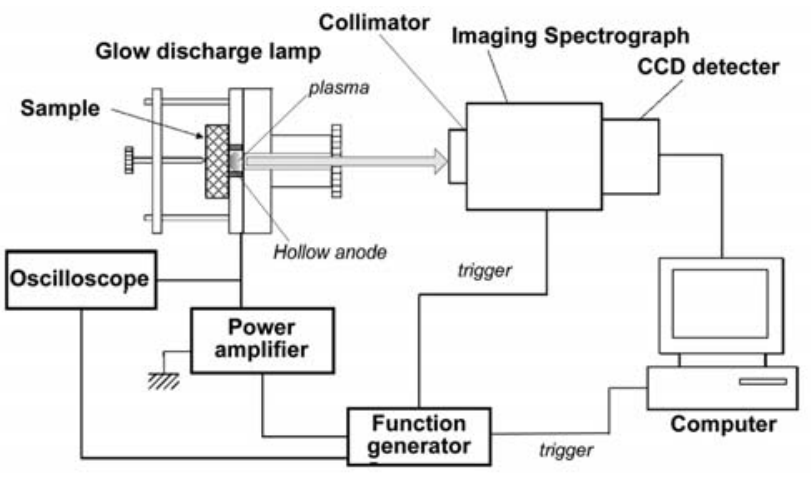

Fig. 1 Block diagram of the measuring system. 

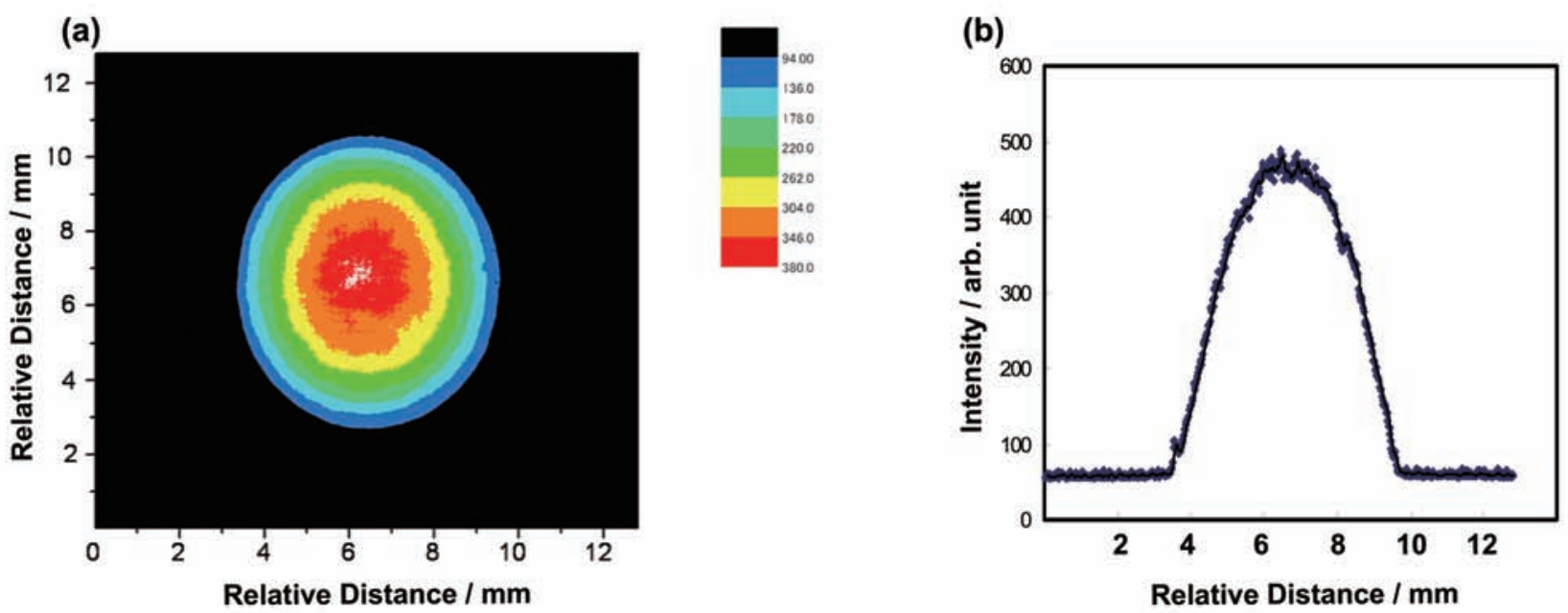

Fig. 2 Two-dimensional emission image of the Zn I 334.50-nm line observed from the axial direction of a glow discharge plasma (a) and the cross-section distribution of the emission intensity along the radial distance (b).

discharge parameters are described later.

High-purity argon (>99.999\%) was introduced as the plasma gas after evacuating the chamber to below $10 \mathrm{~Pa}$. The pressure was monitored with a Pirani gauge (GP-2, ULVAC Corp., Japan), which had been corrected for pure argon, which were placed between the evacuation port and a rotary vacuum pump (GLD-166, ULVAC Engineering Inc., Japan). The plasma gas was flowed during the measurement on keeping a chamber pressure of $5.3 \times 10^{2} \mathrm{~Pa}$.

A pure zinc plate and a pure copper plate were used as the sample. The sample plates were polished with water-proof abrasive papers and then fixed at the sample port of the chamber. After the surfaces were cleaned during pre-discharges, the emission signals were accumulated on a personal computer by using appropriate discharge and measuring conditions for each sample. The analytical lines were $\mathrm{Zn}$ I $334.50 \mathrm{~nm}(7.78 \mathrm{eV})$ and $\mathrm{Cu}$ I $324.75 \mathrm{~nm}(3.82 \mathrm{eV}){ }^{8}$

\section{Results and Discussion}

Figure 2 shows a 2D emission image (a) for the zinc sample, whose intensities are expressed by mapping with several colors, and the cross-section distribution (b) along the radial distance of the plasma. For this measurement, a pulsated discharge mode was employed, ${ }^{9,10}$ where the frequency of the discharge was 100 $\mathrm{Hz}$, the duty ratio $10 \%$, the maximum voltage $450 \mathrm{~V}$, and the minimum voltage (offset voltage) $50 \mathrm{~V}$. The pulsated discharge is required to accumulate the emission image in the CCD detector, and it is also useful to control the sputtering rate appropriately. ${ }^{9}$ The slit width of the spectrometer was $2.0 \mathrm{~mm}$ and the gate width of the CCD detector was $1 \mathrm{~ms}$. The emission signal was stored in the CCD detector during 32 discharge pulses, and this accumulation was 100-times replicated and averaged on the personal computer. Figure 3 shows a $2 \mathrm{D}$ emission image for the copper sample. The following discharge conditions were employed: a maximum voltage of $500 \mathrm{~V}$ and a minimum voltage of $100 \mathrm{~V}$, and the same frequency and duty ratio as the zinc sample. In this case, the slit width was $4.0 \mathrm{~mm}$, the CCD gate width was $1 \mathrm{~ms}$, and the accumulation times were set to be 200 .

These images became a little enlarged in the vertical direction,

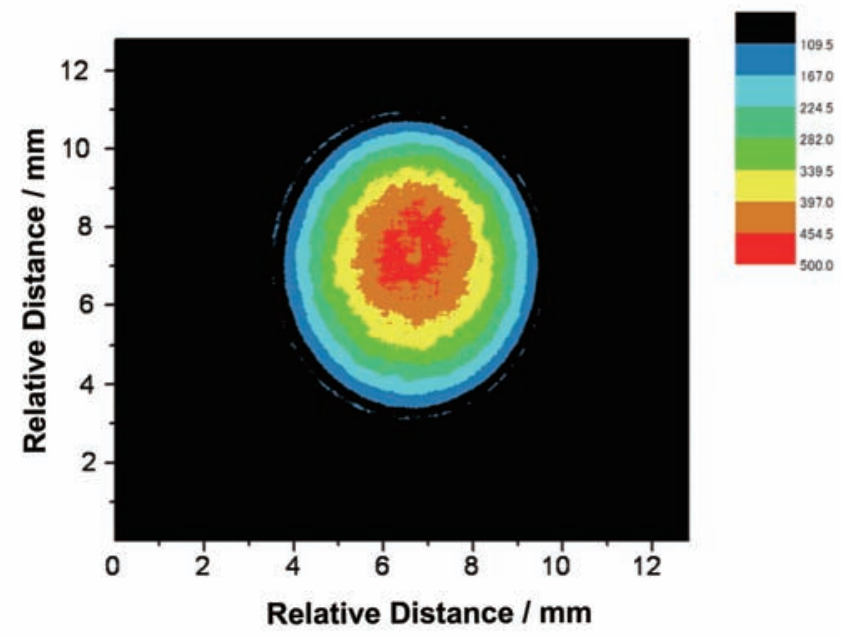

Fig. 3 Two-dimensional emission image of the $\mathrm{Cu}$ I 324.75-nm line.

which might be caused by any aberration through the slit of the spectrograph. It is found that these emission images are similar, being less dependent on the kind of the cathode sample, which indicates that the $2 \mathrm{D}$ intensity image could be determined by the plasma characteristics. Note that the diameter of the hollow cathode is $8.0 \mathrm{~mm}$; therefore, this diameter restricts the plasma zone. It can be clearly demonstrated that the emission of the samples is not irradiated uniformly over the possible plasma zone but has a large variation in the intensities along the radial distance. The central portion having a diameter of about $2 \mathrm{~mm}$ gives the most intense emission. The concentric-circles pattern means that the emission intensity becomes weaker with increasing the diameter. This effect is probably because plasma parameters determining the excitation processes, such as electron density and electron temperature, might be changed in the radial direction of the glow discharge plasma. We are not surprised at this result, because the plasma region should reach a boundary near the hollow anode (anode dark space) ${ }^{11}$ and the resulting emission is thus extinguished. However, the shape of the emission zone and the intensity distribution have not been 
reported in previous papers. This result could provide nove information for understanding the excitations occurring in the glow discharge plasma. We also expect that such spatiallyresolved measurements could contribute to the actual application in GD-OES.

\section{Acknowledgements}

The authors gratefully acknowledge the financial support by a grant from the Ministry of Environment, Japan. This research is supported also by Grant-in-Aids from the Ministry of Education, Science, Sports and Culture of Japan (No. 18360016).

\section{References}

1. R. Payling, D. G. Jones, and A. Bengtson (ed.), "Glow Discharge Optical Emission Spectrometry", 1997, John
Wiley \& Sons, Chichester.

2. K. Marcus and J. A. C. Broekaert (ed.), "Glow Discharge Plasmas in Analytical Spectroscopy", 2003, John Wiley \& Sons, Chichester.

3. K. Wagatsuma, K. Kodama, and H. Park, Anal. Chim. Acta, 2004, 502, 257.

4. H. Yasuhara, A. Yamamoto, K. Wagatsuma, and F. Hiramoto, ISIJ Int., 2006, 46, 1054.

5. G. Gamez, S. J. Ray, F. J. Andrade, M. R. Webb, and G. M. Hieftje, Anal. Chem., 2007, 79, 1317.

6. W. Grimm, Spectrochim. Acta, 1968, 23B, 443.

7. K. Wagatsuma and K. Hirokawa, Surf. Interface Anal., 1986, 8,37 .

8. C. E. Moore, "Atomic Energy Levels", 1952, Vol. 2, NBS Circular 467, Washington D.C.

9. K. Wagatsuma, ISIJ Int., 2004, 44, 108.

10. H. Park and K. Wagatsuma, Anal. Sci., 2006, 22, 599.

11. A. von Engel, "Ionized Gases", 1965, Clarendon Press, Oxford. 